\title{
Workshop Proposal on Knowledge Discovery from Digital Libraries
}

\author{
Hui Shi \\ California State Polytechnic \\ University, Pomona USA \\ huishi@cpp.edu
}

\author{
$\mathrm{Wu} \mathrm{He}$ \\ Old Dominion University, USA \\ whe@odu.edu
}

\author{
Guandong $\mathrm{Xu}$ \\ University of Technology Sydney, \\ Australia \\ guandong.xu@uts.edu.au
}

\section{OVERVIEW}

The workshop is with the ACM/IEEE Joint Conference on Digital Libraries in 2018 (JCDL 2018) which will be held in Fort Worth, Texas, USA on June $3-7,2018$. The Joint Conference on Digital Libraries (JCDL) is a major international forum focusing on digital libraries and associated technical, practical, and social issues.

We solicit contributions on Knowledge Discovery from Digital Libraries. Knowledge is defined as facts, information, descriptions or skills acquired through experience or education. Valid, useful knowledge can help people make better predictions, support decision making and improve people's lives. Knowledge Discovery focuses on searching and extracting useful knowledge from data, databases and documents with different methodologies. The goal of Knowledge Discovery is mainly to uncover hidden relationships between data with techniques from artificial intelligence, mathematics, statistics, and algorithms.

A digital library stores and organizes a collection of digital objects, such as text, images, videos, sounds. Some of the characteristics that digital library has can make knowledge discovery easier: 1) digital library is an open platform. Users from all over the world can have access to the collection of digital library via internet; 2) digital library is an integration of a variety of information and multimedia services which allows multiple access; 3) digital library can provide fast and efficient access and user-friendly interfaces for users to retrieve information; 4) digital library usually is a large, well-organized collection that persist over time. It can contain many formats

\section{ACM Reference format:}

Hui Shi, Wu He, and Guandong Xu. 2018. Workshop Proposal on Knowledge Discovery from Digital Libraries. In Proceedings of The 18th ACM/IEEE foint Conference on Digital Libraries, Fort Worth, Texas USA, fune 3-7 2018 (FCDL'18), 2 pages.

https://doi.org/10.1145/3197026.3200207 and digital objects that may be otherwise unobtainable. All the above characteristics of digital library will facilitate knowledge extraction, transformation, analyzing and presentation.

\section{The objectives of the workshop are to explore:}

1. Advanced ways to organize and maintain Digital Libraries to facilitate Knowledge Discovery

2. Knowledge Discovery applications in Business

3. New challenges and technologies have been brought to the area of knowledge Discovery and Digital Libraries

4. Existing and novel techniques to extract and present knowledge from Digital Libraries

\section{TOPICS OF INTERESTS}

We invite original articles related to Knowledge Discovery from Library and Digital Libraries, which include but are not limited to:

- Information Visualization techniques in Knowledge Discovery from Digital Libraries

- Information extraction and text-mining approaches for semantic enrichment of Digital Libraries

- Ontology driven Knowledge Discovery from Digital Libraries

- Case-based reasoning systems for knowledge discovery from Digital Libraries

- $\quad$ Applications of Sematic Web in Digital Libraries

- Automatic categorization and clustering of scientific publications

- Academic recommender systems

- Applications of mining from scientific publications

\section{SUBMISSION DETAILS}

Each paper submitted to the workshop will be carefully evaluated based on originality, significance, technical soundness, and clarity of exposition and should be prepared according to the JCDL conference Authors Guidelines. It must be no longer than 10 pages and no less than 2 pages in length, including a 200- 
word abstract, references and tables/figures. Authors should submit their manuscripts in PDF format to Dr. Hui Shi (huishi@cpp.edu), Dr. Wu He (whe@odu.edu) and Dr. Guandong Xu (guandong.xu@uts.edu.au) by email. Top quality papers after presented at the conference will be selected for extension and publication in Information Discovery and Delivery published by Emerald.

\section{IMPORTANT DATES}

- Paper Submission: 04/18/2018

- Author Notification: $04 / 30 / 2018$

- Camera-ready Submission (for workshop proceedings): $05 / 15 / 2018$
- Conference Dates: 06/03-06/07/2018

- Workshop Date: 06/06/2018 (half day)

\section{ORGANIZERS}

- Hui Shi, California State Polytechnic University, Pomona, USA

- $\quad \mathrm{Wu}$ He, Old Dominion University, USA

- Guandong Xu, University of Technology Sydney, Australia 